\title{
RECALCITRANT HYPOCALCEMIA IN A PATIENT WITH POST-THYROIDECTOMY HYPOPARATHYROIDISM AND ROUX-EN-Y GASTRIC BYPASS
}

\author{
Gonzalo Allo Miguel, Elena García Fernández, Soledad Librizzi, \\ Guillermo Martínez Díaz-Guerra, Federico Hawkins \\ Endocrinology Service, 12 de Octubre University Hospital.
}

\section{Introduction}

Roux-en-Y gastric bypass (RYGB) places patients at an increased risk of hypocalcemia due to the reduction in calcium absorption (because the procedure bypasses the duodenum and jejunum) and vitamin $D$ deficiency. Subsequent thyroid surgery increases the risk of severe hypocalcemia due to potential post-operative hypoparathyroidism. Only a few cases have been published before of this kind of treatment-challenging hypocalcemia.

\section{Case Report}

We report the case of a 31-year-old woman with a previous RYGB, who suffered severe and symptomatic chronic hypocalcemia after total thyroidectomy. Six months after the surgery, corrected calcium level had severely decreased $(5.2 \mathrm{mg} / \mathrm{dL})$ and the patient related generalized muscle cramps, labile mood and increased anxiety. She required aggressive therapy with oral calcium and calcitriol (higher dose: $12 \mathrm{~g}$ of calcium carbonate and $8 \mu \mathrm{g}$ of calcitriol per day) and frequent calcium infusions, but there was no improvement in serum calcium level. Due to the lack of response to standard therapy, teriparatide treatment was started (first with subcutaneous injections and thereafter with a multipulse subcutaneous infusor) but the results were disappointing. As there was no response to different medical treatments, reversal of RYGB was performed with no complications and a subsequent sustained increase in serum calcium level.

After Roux-en-Y gastric bypass

PTH

个个

Due to a drive to maintain serum calcium levels

\section{$\downarrow$}

Vitamin D Due to fat and vitamind D malabsorption from gastric bypass

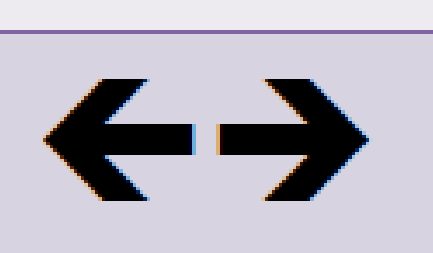

\section{After Thyroid surgery}

\section{$\downarrow$}

Due to stunning of parathyroid glands

\section{$\downarrow$}

Due to fat and vitamind D malabsorption from gastric bypass

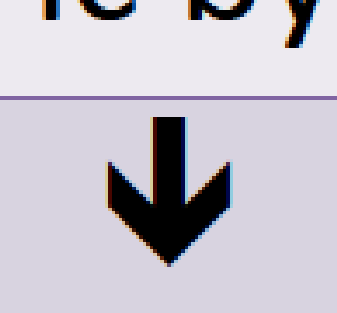

\begin{tabular}{l|c|c} 
Calcium & $\begin{array}{c}\text { Elevated PTH level mantains calcium level despite its } \\
\text { decreased absorption from duodenum and jejunum } \\
\text { bypass }\end{array}$ & $\begin{array}{c}\text { Loss of drive from PTH to maintain calcium } \\
\text { levels and inability to replete with oral calcium } \\
\text { supplementation }\end{array}$ \\
Urinary & $\boldsymbol{\downarrow}$ & $\leftrightarrow$
\end{tabular} Calcium

Elevated PTH level causes decreased renal excretion Loss of drive from PTH causes normalization of of calcium renal calcium excretion

Table 1. Comparison of parathyroid hormone (PTH), vitamin $\mathrm{D}$, calcium and urinary calcium levels after gastric by-pass and after thyroid surgery. Adapted from Megan L. Durr et al'1.

\section{Conclusions}

This case shows that patients with postoperative hypoparathyroidism and RYGB have increased risk of severe recalcitrant symptomatic hypocalcemia. In our case teriparatide was ineffective but, as this is the first patient reported, more results are needed to evaluate properly the effect of teriparatide in this kind of hypocalcemia. The reversal of RYGB may be an optimal treatment, if medical management has failed, because the surgery recovers an adequate absorption of calcium and vitamin $D$ from previously bypassed duodenum and proximal jejunum.

\section{Bibliography}

1. Durr ML, Saunders JR, Califano JA, et al. Severe hypocalcemia complicating thyroid surgery after Roux-en-Y gastric bypass procedure. Arch Otolaryngol Head Neck Surg. 2009 May;135(5):507-10. 